\title{
Dialogue Management for an Automated Multilingual Call Center
}

\author{
Hilda Hardy, Tomek Strzalkowski and Min Wu \\ Institute for Informatics, Logics and Security Studies \\ University at Albany, Albany, NY 12222 \\ and \\ The AMITIES Consortium ${ }^{1}$ \\ hardyh, tomek, minwu@cs.albany .edu
}

\section{Introduction}

The AMITIÉS project (Automated Multilingual Interaction with Information and Services) has been established under joint funding from the European Commission's $5^{\text {th }}$ Framework Program and the U.S. DARPA to develop the next generation of empiricallyinduced human-computer interaction capabilities in spoken language. One of the central goals of this project is to create a dialogue management system capable of engaging the user in human-like conversation within a specific domain. The domain we selected is telephonebased customer service where the system has access to an appropriate information database to support callers' information needs. Our objective is to automate at least some of the more mundane human functions in customer service call centers, but do so in a manner that is maximally responsive to the customer. This practically eliminates all prompt or menu based voice response systems used at commercial call centers today.

Exploiting the corpus of hundreds (and soon to be thousands) of annotated dialogues, recorded at European financial call centers, we have developed a call triaging prototype for financial services domain. This demonstrator system handles the initial portion of a customer call: identifying the customer (based on a sample customer database) and determining the reason the customer is calling (based on a subset of transactions handled at the call center). Our approach to dialogue act semantics allows for mixed system/customer initiative and spontaneous conversation to occur. We are currently extending this prototype beyond its triage role to negotiate and execute the transactions requested by the customers, ranging from simple address changes to more complex account payment transactions.

The aim of AMITIES project is to build a largescale, empirical system using data-driven design, derived from actual and purposeful (i.e., not acted or contrived) human-to-human dialogues. This proves to be a lengthy and complicated process due to a variety of le- gal constraints we need to overcome to obtain real data in sufficient quantities. We have devoted a considerable effort to this issue, which only now is beginning to bring results. The prototype described here has not been empirically validated yet.

\section{Dialogue with Information and Services}

The key concept underlying AMITIES dialogue manager is the notion of dialogue with data. The prevalent type of dialogue in a call center environment is information seeking/information access, which displays specific characteristics that can be exploited in the design of an automated system. In a human-operated call center, an operator mediates between the caller and a variety of data sources: information about customers, products, regulations, etc. Much of this data is in a structured form, usually a relational database (accounts information), while some may remain in an unstructured form (e.g., text memos, flyers, regulations manuals.) The objective of an automated call center is to obtain a naturally interactive mediation, between the caller and the information which is as close to a human-human dialogue as possible.

This automated call center scenario applies to many customer service situations, including the following:

- Financial services (AMITIES primary domain)

- Product support

- Travel reservations

where the objective is to locate, insert or update a single (or several) data object in a structured data base. At a more abstract level, the call center of the type described here can be characterized as an Interaction with Structured Data (ISD). ISD consists of the following components:

1. Data structure, which defines the set of basic entities (accounts, spare parts, flights) and their attributes (account number, part size, destination city, etc.) as well as methods for identifying references to these attributes in user statements.

\footnotetext{
${ }^{1}$ The AMITIES consortium members include University of Sheffield, CNRS-LIMSI, Duke University, SUNY Albany, VESCYS, and Viel et Cie.
} 
2. List of basic transactions supported by the service (account payment, address change, locating a flight) along with methods to detect references to these transactions.

3. Dialogue models for handling various conversational situations in human-like fashion (e.g., responding to requests, emotions, indecision) and consistent with the character of the service (polite, helpful, caring).

4. Optional dialogue meta-strategy as required to address privacy and security concerns (e.g., positive caller identification must precede exchange of any sensitive information.)

The components 1,2 and 4 can be built using limited amount of static data about the service and are to a large degree domain-independent or domain-adaptable. These components are sufficient to design basic mixedinitiative dialogue capabilities, as explained further in the following section. Although the dialogue may not feel very "natural" it will be quite efficient, giving the user a broad initiative to conduct it as they wish. Dialogue models (component \#3) are required to create an illusion of naturalness and these can only be derived from large corpora of actual call center conversations. Large corpora of real conversations are also needed to develop speech and prosody models.

We have built a prototype caller triaging dialogue management which has been incorporated in the first AMITIES demonstrator. The system is based on Galaxy Communicator architecture (Seneff et al., 1998) in a standard configuration shown in Figure 1. The DM can handle dialogues in 3 European languages, and can additionally switch from one language to another in midconversation.

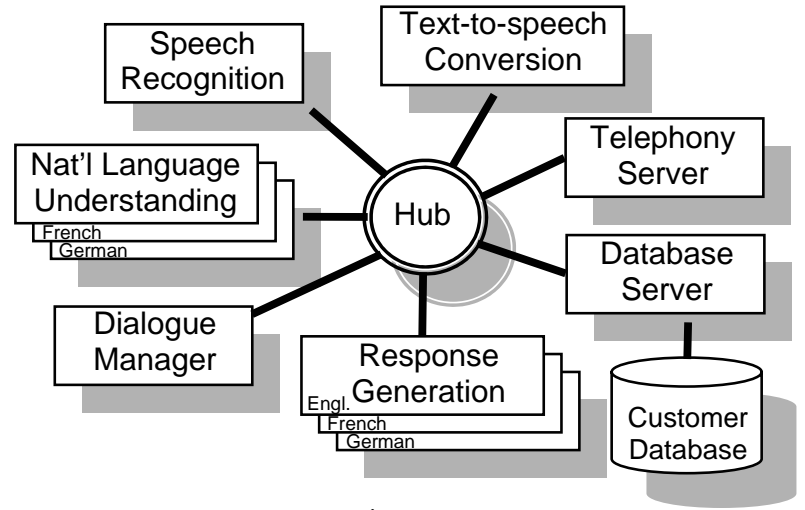

Figure 1. AMITIÉS System Architecture

\section{Dialogue Manager/Frame Router}

In this section we explain some key principles of designing an interactive dialogue with Structured Data (ISD). The overall strategy is to locate an item or items in the database that meet a number of specific conditions, for example, the most convenient flight, the caller's bank account, etc. This overall objective is bro- ken down into a set of sub-goals some of which may need to be satisfied to achieve the objective. The role of ISD dialogue is to chart a path through the sub-goals in such as way that:

1. the objective is achieved

2. any partial constraints on the order or selection of the sub-goals are met, and

3 . the most efficient route is chosen.

The dialogue manager identifies the goal of the conversation and performs interactions to achieve that goal. The overall mechanism works by filling attribute values in frames representing transactions and the sub-goals. Spontaneous conversation works in this environment, because values may be filled in any order, or several values may be supplied in one turn. As attribute values in the frames are filled, the need for dialogue decreases.

The system sets key milestones or goals to be reached by gathering sufficient information from the customer, but these milestones may be approached by a variety of different paths. If the customer's last name is misrecognized, for example, or if there are multiple database records returned, the system will ask for a different attribute, such as the address or postal code. Reprompts are used when necessary, but no more than once for any single attribute. The process continues until a unique (e.g., bank account) or best (e.g., a flight) record is identified. Thus the dialogue system has flexibility to deal with user input arriving in any order or form and the input that is not completely captured, without getting stuck on any single attribute. The paths to the key milestones, and even the order of the milestones, may be seen as a series of hidden transitions. This means exact progression of the dialogue is never pre-set or can be known in advance - a major advance over system-driven prompts.

In order to keep the dialogue manager language- and domain-independent, mechanisms were created to store the language-specific and task-specific information in separate modules, to be loaded as needed. These are illustrated in Figure 2.

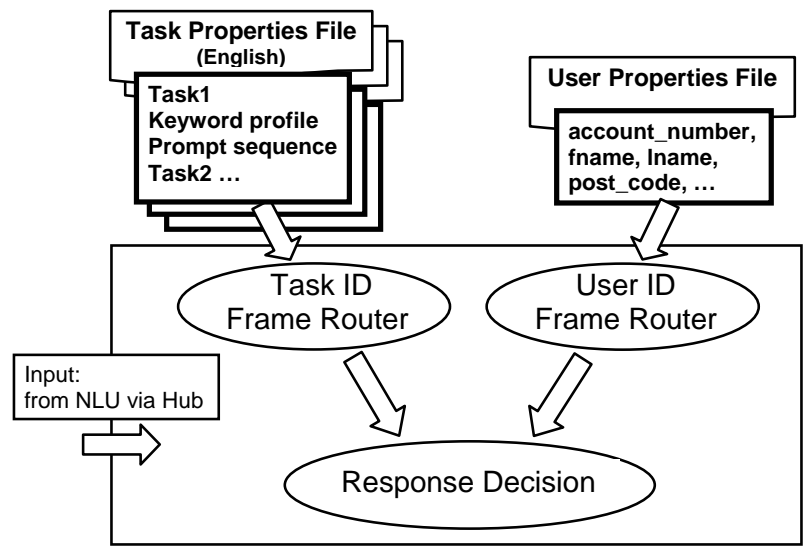

Figure 2. Dialogue Manager Structure 
In Fig. 2, the transaction identification module (TaskID Frame Router) matches the incoming user utterances to identify which transaction is being invoked. If multiple transactions are matched, their representations (frames) are ranked in the likelihood order. Each frame consists of a keyword profile (a list of salient terms derived from human-human dialogues) and a prompt sequence, which is a list of attributes whose values must be collected in order to complete the transaction. This prompt sequence is passed to the Dialogue Manager which then handles it in a manner consistent with the adopted dialogue model. Task identification succeeds even with high speech recognition error rates, provided the keywords are detected accurately. ${ }^{2}$ Ties (or near ties) are resolved through clarification dialogue by determining the value of some contrasting attribute, which is dynamically selected for each situation. The User ID Frame Router operates analogously, with each frame representing a different user (customer).

Two simple dialogues with AMITIES are below:

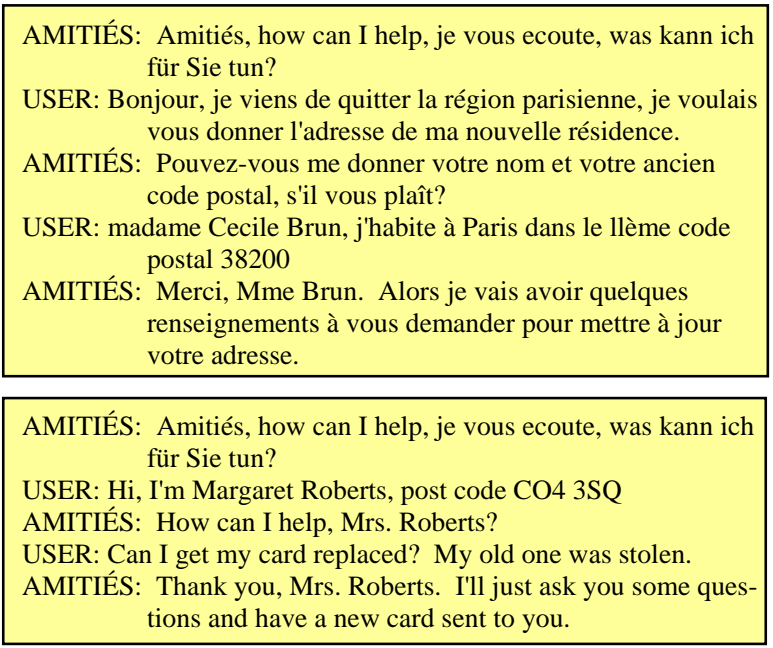

\section{Dialogue Annotations}

Working with the real call center dialogues required us to develop a new method for dialogue annotation. The DAMSL functional annotation scheme had to be modified and further augmented with semantic annotation. To do so, we have created dialogue act taxonomy appropriate for ISD dialogues. To capture the semantics, we used a domain-independent framework populated with domain-specific lists. Furthermore, to facilitate speedy annotation, we have designed a new flexible, annotation tool, XDMLTool, and annotated several hundred French and English dialogues using it.

In order to annotate semantic information with XDMLTool, the user makes entries for a particular turn or turn segment in a semantic table on the user interface.

\footnotetext{
${ }^{2}$ While different combinations of keywords may invoke a transaction frame, this process is robust because the selection of transactions is limited to those known to the system.
}

Transactions such as MAKEPYMNT or CHANGEADDR are selected and their attributes appear in combo-boxes on the GUI. If necessary, the user may type in new labels. To fill a value for an attribute, text from the displayed dialogue may be copied into a table cell.

For example, the following exchange, part of a VERIFYID transaction, would be labeled with the attributes Name and PostCode. The values John Smith and $A B 11 C D$ would be tagged for the answer.

\section{A: Your full name and postcode please? \\ C: Yes it's err John Smith AB1 1CD}

The new annotation scheme reflects our approach to dialogue design - we hope it will help us to automatically derive appropriate dialogue strategies for novel ISD situations, and beyond. ${ }^{3}$

\section{Acknowledgments}

This paper is based on work supported in part by the European Commission under the $5^{\text {th }}$ Framework IST/HLT Programme, and by the U.S. Defense Advanced Research Projects Agency.

\section{References}

J. Allen and M. Core. 1997. Draft of DAMSL: Dialog Act Markup in Several Layers. http://www.cs. rochester. edu/research/cisd/resources/damsl/.

J. Allen, et al. 1995. The TRAINS Project: A Case Study in Building a Conversational Planning Agent. Journal of Experimental and Theoretical AI, 7, 7-48.

AMITIÉS, http://www.dcs.shef.ac.uk/nlp/amities/.

A. Bagga, T. Strzalkowski and G. B. Wise. 2000. Parts ID : A Dialogue-Based System for Finding Parts for Medical Systems. In Proc. of ANLP-2000.

J. Chu-Carroll and B. Carpenter. 1999. Vector-Based Natural Language Call Routing. Computational Linguistics, 25 (3): 361-388.

DARPA, http://www.darpa.mil/iao/Communicator.htm.

L. Devillers, S. Rosset, H. Maynard and L. Lamel. May 2002. Annotations for Dynamic Diagnosis of the Dialog State. In Proc. of LREC, Las Palmas.

R. Gaizauskas et al. 1996. GATE : An Environment to Support Research and Development in Natural Language Engineering. In Proc. Of 8th IEEE Int. Conf. on Tools with AI, Toulouse, France.

A. L. Gorin, G. Riccardi and J. Wright. 1997. How May I Help You? Speech Comm., 23 (1/2): 113-127.

S. Seneff, E et al. 1998. Galaxy-II: A Reference Architecture for Conversational System Development. In Proc. of ICSLP 98, Sydney, Australia.

\footnotetext{
${ }^{3}$ Some preliminary results of dialogue structure analysis are available but we lack space to include them in this note.
} 